\title{
Fractal Dimension of a Coral Reef at Ecological Scales
}

\author{
R. H. Bradbury and R. E. Reichelt \\ Australian Institute of Marine Science, P.M.B. No. 3, MSO Townsville, Queensland 4810, Australia
}

\begin{abstract}
Conventional analytical techniques do not cope adequately with multiscale processes. Mandelbrot's concept of fractal dimension, a novel approach to multiscale phenomena, is applied to the problem of coral reef topography. A fractal dimension $D=1.9-2.0$ for a contour on the reef slope is obtained. This contrasts strongly with the well established value of $D=1.2-1.3$ for coastlines. We speculate that in common with the human lung, another living surface with a high $D$ value, the coral reef slope is maximizing its contact with the surrounding medium.
\end{abstract}

\section{INTRODUCTION}

Coral reefs are complex systems (Grassle, 1973). Our attempts to understand them necessarily involve comprehension of their complexity, usually through the intercession of mathematics. One strategy is to so simplify our conceptual model of the coral reef that the complexity is reduced to a level tractable with conventional analytical techniques. But it has been argued elsewhere (Bradbury and Young, 1981) that it is the complexity itself that is the distinguishing feature of coral reefs. If this is so, the strategy of simplification cannot address the interesting fundamental questions about coral reefs as systems, and another strategy is called for.

This other strategy is the one we shall adopt here. It allows the innate complexity of the system full rein and tackles it directly by appropriate, if novel, techniques.

The particular aspect of coral reef complexity that interests us here is this: Some important processes appear to operate simultaneously over many physical scales and biological levels of organization (Bradbury and Loya, 1978). Our understanding and therefore analysis of such processes need somehow to be integrated over these scales or levels for them to be useful.

Coral reef topography is a critical example of this 'complexity at many scales'. Early attempts at its measurement ignored its characteristic multiscale aspect and therefore produced trivial interpretations of its ecological significance (Talbot and Goldman, 1972;
Luckhurst and Luckhust, 1978). We aim, in this report, to investigate the applicability of the concept of fractal dimension to this previously intractable phenomenon. We seek to show that the concept might provide useful ecological insights while retaining the full complexity of the multiscale nature of the phenomenon. We will argue that the technique holds promise and that it warrants further exploration.

\section{CONCEPT OF FRACTAL DIMENSION}

Mandelbrot (1977) coined the term 'fractal' for a series that is continuous yet nondifferentiable. A continuous series, such as a polynomial, is differentiable because it can be split up into an infinite number of absolutely smooth straight lines. Each splitting process on a fractal series, by contrast, reveals nonlinear structures which themselves require resolution. For curves, whose topological dimension is always 1 , the fractal dimension $D$ can vary between 1 and 2 . When $D=1$, the curve is completely differentiable and does not have fractal properties. As D approaches 2, the curve becomes increasingly convoluted at all scales. It is not differentiable and in the limit fills the plane. Thus the fractal dimension of a fractal series always exceeds its topological dimension. The same ideas apply to planes where the topological dimension is 2 and the fractal dimension is $2<\mathrm{D}<3$. The present study is concerned with the fractal properties of curves. 


\section{ANALYTICAL METHODS}

The fractal dimension of a theoretical fractal set may be derived analytically, and many examples are given in Mandelbrot (1977). For an empirical data set the derivation usually involves fitting the empirical data to a theoretical set of known fractal dimension. Again, Mandelbrot gives many examples of such attempts. The straightforward solution for a curve involves enumerating the empirical set at the smallest possible scale over the largest possible scale. This is equivalent to drawing the curve with the greatest possible detail over its whole length. Clearly, this is not often possible in practice. We have chosen instead to estimate the fractal dimension by following Burrough (1981).

He noted that the Weierstrass-Mandelbrot fractal function W (t), as explicated by Berry and Lewis (1981), has a variance of increments

$$
V(t)=\left\langle\left[W\left(t_{0}\right)-W\left(t_{o}+t\right)\right]\right\rangle
$$

that varies as $t^{4-2 D}$ at the origin. Thus we may estimate the fractal dimension $D$ of a real series from

$$
\frac{d \log \mathrm{V}(\mathrm{t})}{d \log \mathrm{t}}=4-2 \mathrm{D}(\mathrm{t} \rightarrow \mathrm{O})
$$

Burrough also noted that the variance of increments is frequently used in geostatistical studies where it is called the variogram function (Journel and Huijbregts, 1978) and, there, empirical techniques to estimate it are common. We have adapted these for the coral reef. In particular we have looked at the problem over a greater range of scales than is the case in geostatistics. Our spatial scales range over 3 orders of magnitude, whereas geostatistical studies usually straddle only 1 . Sales and Thomas (1978) show that many studies of topography from a wide range of surfaces cover only 1 or 2 magnitudes.

\section{FIELD METHODS}

We chose the contour at $2 \mathrm{~m}$ depth on the windward reef slope of Myrmidon Reef $\left(18^{\circ} 20^{\prime} \mathrm{S}, 147^{\circ} 20^{\prime} \mathrm{E}\right)$ as the curve whose fractal dimension we wished to estimate.

The basic measurements were made as follows. A step of length $l$ was made along the contour by following the surface as closely as possible. The step touched the contour at its two ends, straddling concavities and intersecting convexities in the surface. At the beginning of this step, a series of similar but smaller steps was begun. These measured $1 / 101$. The number of these smaller steps that fitted within each larger step is our measure whose variance was used to estimate $D$.
This procedure was repeated at each of a series of length $I$ to cover a range of scales of ecological interest. The lengths chosen were $10 \mathrm{~cm}, 1 \mathrm{~m}, 10 \mathrm{~m}$ and $100 \mathrm{~m}$. For each scale, 25 steps were made along the contour, giving 25 replicate measures with which to estimate the variance. The $10 \mathrm{~cm}, 1 \mathrm{~m}$ and $10 \mathrm{~m}$ ranges were measured directly using large, specially constructed geometer's dividers. The $100 \mathrm{~m}$ range was measured on an aerial photograph, where the $2 \mathrm{~m}$ depth contour was reasonably obvious. At the $10 \mathrm{~cm}$ range, the steps were not contiguous since a few large adjacent corals of one type could have biased our estimate. Instead 25 nonoverlapping replicates were randomly located within a $10 \mathrm{~m}$ segment of the contour.

\section{RESULTS}

Table 1 summarizes our estimates of the measure and its variance at each of the scales. The log-log plot of variance against scale is shown in Fig. 1 with a leastsquares fit superimposed. The slope of this line, 0.046 , equals $4-2 \mathrm{D}$ as indicated in Equation 2. Thus the fractal dimension $\mathrm{D}$ is approximately 1.9-2.0.

It should be emphasized that this is an approximate result, since the method is under development. We conclude no more than that the value of D lies near 2 .

Table 1. Results of step length counts along $2 \mathrm{~m}$ contour on Myrmidon reef

\begin{tabular}{|lcccc|}
\hline Step length $l$ & $10 \mathrm{~cm}$ & $1 \mathrm{~m}$ & $10 \mathrm{~m}$ & $100 \mathrm{~m}$ \\
\hline $\begin{array}{l}\text { Number of replicates } \\
\text { Mean number of sub }\end{array}$ & 25 & 25 & 25 & 25 \\
$\begin{array}{l}\text { steps per step length } \\
\text { Variance }\end{array}$ & 14.4 & 14.6 & 14.4 & 13.4 \\
\hline
\end{tabular}

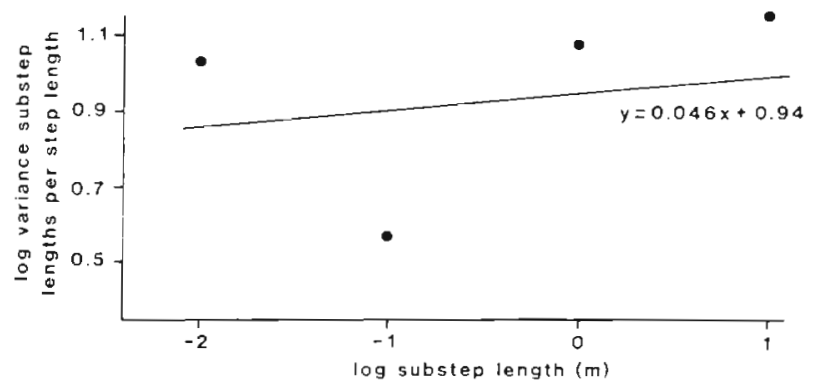

Fig. 1. Plot of $\log$ variance of the number of substep lengths per step length against log substep length in $m$ 


\section{DISCUSSION}

Mandelbrot (1977) amassed considerable data to show that the fractal dimensions of coastlines and other linear natural phenomena are in the range $\mathrm{D}=$ $1.2-1.3$. Our result, $\mathrm{D}=1.9-2.0$, suggests that the coral reef surface is much more complex and that this complexity extends over several orders of magnitude.

But what is the ecological significance of this result? What do high or low values of D signify for a coral reef? The beginnings of an answer require an understanding of how curves change as D increases. Consider a curve in a plane to divide the plane into 2 phases, say inside and outside the curve. When D is 1 , the curve is not a fractal and fully differentiable. Points may be found inside the curve which are far from points outside the curve. As D increases, the convolutions in the curve increase at all scales even though the area of the 2 phases remains the same. Thus points inside the curve become closer on average to points outside the curve. As $D$ aproaches 2, the curve becomes so convoluted that it tends to fill the space, even though the areas of the 2 phases have not changed. Thus in the limit, the distance between any point inside or outside the curve and the curve itself vanishes; that is, every point lies on the surface of the curve.

Mandelbrot has shown that a two-dimensional model of the lung has a $\mathrm{D} \rightarrow 2$, and the property that every point in one phase (air or blood) is infinitely close to a point in the other phase.

The similarity of our result to the two-dimensional lung model rather than the coastline model suggests to us that coral reefs are living surfaces which attempt to come into as intimate and complete contact as possible with the surrounding medium. Thus the chance is maximized that any part of the water moving over the reef comes in contact with a part of the living surface. Conversely, the chance is also maximized that any part of the living surface comes in contact with a part of the water.

Myrmidon reef lies on the edge of the continental shelf adjacent to the oceanic water of the Coral Sea. A high value of $D$ may be an efficient means for such a reef ecosystem to cope with oceanic waters with low levels of particulate organic matter (Andrews and Gentien, 1982). We might expect different values of $D$ to be found in inshore reefs where the water is generally richer.
We might alo expect reef slopes, which are the first parts of a reef to meet the medium, to have different values of $D$ from, say, reef flats or back reef areas where the need may be to reduce the intimacy of contact with the medium in order to retain products within the ecosystem.

It could also be argued that at scales above and below those studied here, the values of $\mathrm{D}$ might be different, since the system might be behaving differently at different scales. Parenthetically, the scatter of points in Fig. 1 is an indicator that the fractal dimension may not be uniform even over the scales we have measured. In fact, Mandelbrot (1977) has marshalled some arguments to suggest that some real fractal sets show just this behaviour.

These ideas suggest further studies to refine the method and to measure different reefs. Even so, it is likely that the concept of fractal dimension has much to offer the study of complex ecosystems such as coral reefs.

\section{LITERATURE CITED}

Andrews, J. C., Gentien, P. (1982). Upwelling as a source of nutrients for The Great Barrier Reef ecosystems: a solution to Darwin's question? Mar. Ecol. Prog. Ser. 8: 257-269

Berry, M. V., Lewis, Z. V. (1980). On the Weierstress-Mandelbrot fractal function. Proc. R. Soc. Lond. A 370: 459-484

Bradbury, R. H., Loya, Y. (1978). A heuristic analysis of spatial patterns of hermatypic corals at Eilat, Red Sea. Am. Nat. 112: 493-507

Bradbury, R. H., Young, P. C. (1981). The effects of a major forcing function, wave energy, on a coral reef ecosystem. Mar. Ecol. Prog. Ser. 5: 229-241

Burrough, P. A. (1981). Fractal dimensions of landscapes and other environmental data. Nature, Lond. 294: 240-242

Grassle, J. F. (1973). Variety in coral reef communities. In: Jones, O. A., Endean, R. (eds.) Biology and geology of coral reefs, Vol. 2, Biology 1. Academic Press, New York, pp. $247-270$

Journel, A. G., Huijbregts, Ch. J. (1978). Mining geostatistics. New York, Academic Press

Luckhurst, B. E., Luckhurst, K. (1978). Analysis of the influence of substrate variables on coral reef fish communities. Mar. Biol. 49: 317-323

Mandelbrot, B. (1977). Fractal form, chance and dimension, San Francisco, Freeman

Sales, R. S., Thomas, T. R. (1978). Surface topography as a nonstationary random process. Nature, Lond. 271: $431-434$

Talbot, F. H., Goldman, B. (1972). A preliminary report on the diversity and feeding relationships of the reef fishes of One Tree Island, Great Barrier Reef System. Proc. 1st Int. Symp. Coral Reefs: $425-443$ 